\title{
The Study of Configuration Analysis in Art Gallery based on Space Syntax
}

\section{-Comparison of Washington national gallery and the Shanghai art gallery}

\author{
Fei Qi ${ }^{1, a}$, Yi Chen ${ }^{2, b}$ \\ ${ }^{1}$ No 1239 , Siping road, ShangHai, China \\ ${ }^{2}$ No 1239 , Siping road, ShangHai, China \\ aifei@tongji.edu.cn, ${ }^{\text {b }}$ chenyi@tongji.edu.cn
}

\begin{abstract}
Keywords: Spatial configuration, Art gallery, Space syntax
Abstract. The article provides detailed and accurate design basis for building plan, studies a new theory and technology for the designers by researching from the spatial essentials to mathematically logic of spatial configuration and by using the method of spatially quantitative description, such as convex space, all-line visibility map, etc. The west part of national art gallery in Washington DC and Shanghai art gallery are taken as examples for analyzing spatially variable visibility and describe local space configuration and configurational relationship.
\end{abstract}

\section{Spatial configuration of gallery}

The main function of the art gallery is to inform the public of new knowledge, not only by providing valuable material and cultural experiences on the certain places or certain times, but also by paying attention to the space for public assembly. Art gallery, generally as a clear organizational structure, is composed of the exhibition hall devoted to display various art forms such as painting, sculpture, installation, furniture and multimedia, etc, the art education area, the management space or office department and artwork store. On social functionally, art gallery talks about stories of history, environment and humanities in a narrative way. Artwork, to a certain extent, is a microcosm of human life, the layout of art gallery often changes according to continuously changing exhibition themes, the space configuration will also change, different spatial configuration forms a specific person assembled mode and different interactive mode. Some studies posit concrete discussion about conceptual shifts during design with particular emphasis on the designer's understanding of what kind of configuration the particular design is(Peponis, Bafna et al. 2015) ${ }^{[1]}$. Similar to a small social community, art gallery is the architecture about the spatial organization with the precise organizational structure and has a great difference from other building types. Tzortzi, Kali ${ }^{[2]}$ argued that it is possible to distinguish between art galleries that intend to convey a pre-given meaning and reproduce information and art galleries that aim at creating fields of possible meaning and producing a richer spatial structure.

Art gallery plan, as a carrier of narrative function, on one hand, to guide visitors and form visit flow line, however, different visitors will have different flow lines, because the different choice concerning to route sequence and the exhibit we had encountered and the impression which we have been experienced in our memory; on the other hand, art gallery plan can give visitors indications, so that visitors who are familiar with the local area in the visiting process can understood within a larger overall environment as soon as possible and are unlikely to lost in larger galleries. J. Peponis ${ }^{\text {[3] }}$ provides overall statement by offering a computational description and provisional statistical profile of properties of building layouts that contribute to making them intelligible.

Yoon Kyung Choi ${ }^{[4]}$ mentioned in his study: "art galleries seem particularly interesting, because it is mainly applied exploratory mode, its functional organizations are relevant to what curator want to display, functional organization on the floor plan also determines the encounter pattern between the 
visitors and the exhibit objects. For visitors, walking in art gallery not only in manner of empirical cumulatively knowledge, but also with some kinds of adventure experiences, it is completely dependent on the overall pattern and intelligibility plan of integration".

By quantified study of spatial configuration, professional designers or architects can more clearly understand the relationship between human behavior and the built environment, predict human activity and how people use the environment and provide advices and assessments as reference to architecture design.

\section{Washington national art gallery, Shanghai art gallery overview}

\section{Washington national gallery profile}

Washington national gallery, located in Washington D.C., designed by John Russell Pope in 1936, is the most delicate building in the world and one of the art galleries with the richest collection of more than 40,000 pieces, ranging from European middle ages to modern times, from American colonial era to the modern period; the expansion portion had been built on the eastern side of the original building in 1978, known as East Hall, designed by IM Pei who is a noble Chinese-American modern architect master; therefore, the old building is called the West Hall, West Hall with a neo-classical facade of the building strictly follow the principle of axial symmetry on the layout arrangement (Figure 1).

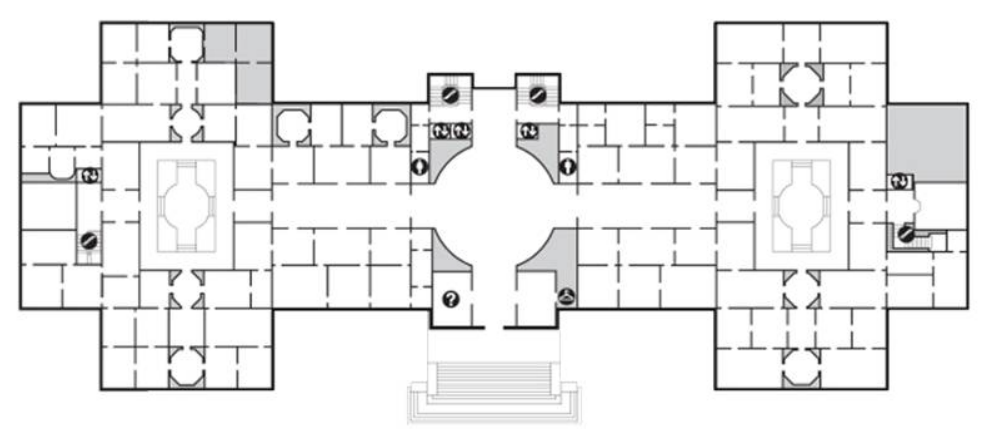

Fig. 1: Main floor plan of Washington national gallery Source: http: //www.nga.gov

\section{Shanghai art gallery profile}

The building, previously the old Shanghai Race Club, was built in 1933 and renovated in 1983. The renovation project totally retained the integrity of its original neo-classical characteristic facade. Shanghai art gallery has a collection of more than 8,000 pieces of artwork ranging from modern and contemporary works of famous artists to local characteristics artworks of paintings, prints, comic books and other artworks, Figure 2 shows floors plan of Shanghai art gallery. The survey coincided with the Shanghai Biennale, the floor plan was separated to make a rearrangement in accordance with the "City Quick" exhibition, the host set a higher exhibition wall for multimedia presentations, small rooms with a top surface and a large scale installation works, etc.
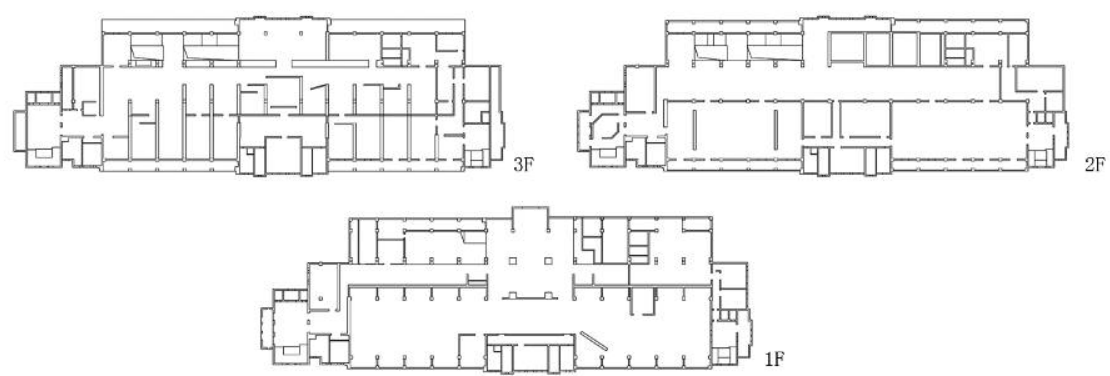

Fig. 2: Shanghai art gallery ground floor to 3rd floor plan Source: "City Quick" exhibition guide map 


\section{Quantitative analysis of spatial configuration}

\section{Comparison of topological depth}

Figure 3 (a) is J-graph of the main floor of Washington national gallery of West Hall, which illustrates the relationship among each of convex spaces from the outdoor as a starting point to the most deepest spaces as end points, you can see that the J-graph of main plan is more complex than the first floor of Shanghai art gallery, with respect to not only more divided convex space, but also a deeper depth level of topology. Seen from the diagram, the Washington national gallery convex spatial distribution is very similar to that of Shanghai art gallery, the difference is that the former with denser convex spatial distribution and more topological depth, average depth of convex space of Washington national gallery is 7.25, is higher than the average depth of Shanghai art gallery on $1^{\text {st }}$ floor plan, convex spatial distribution of the latter is relatively simple and relatively low in topological depth. In turn, by comparing with the floor plan we can prove that floor plan with more spatial quantity and more complex distribution arrangement, its' total topological depth and average topological depth is higher than that of floor plan with a small amount of spaces and the rather simple distribution of convex space, in another words, the larger topological depth of a floor plan is more difficult to recognize than a floor plan with smaller topological depth.
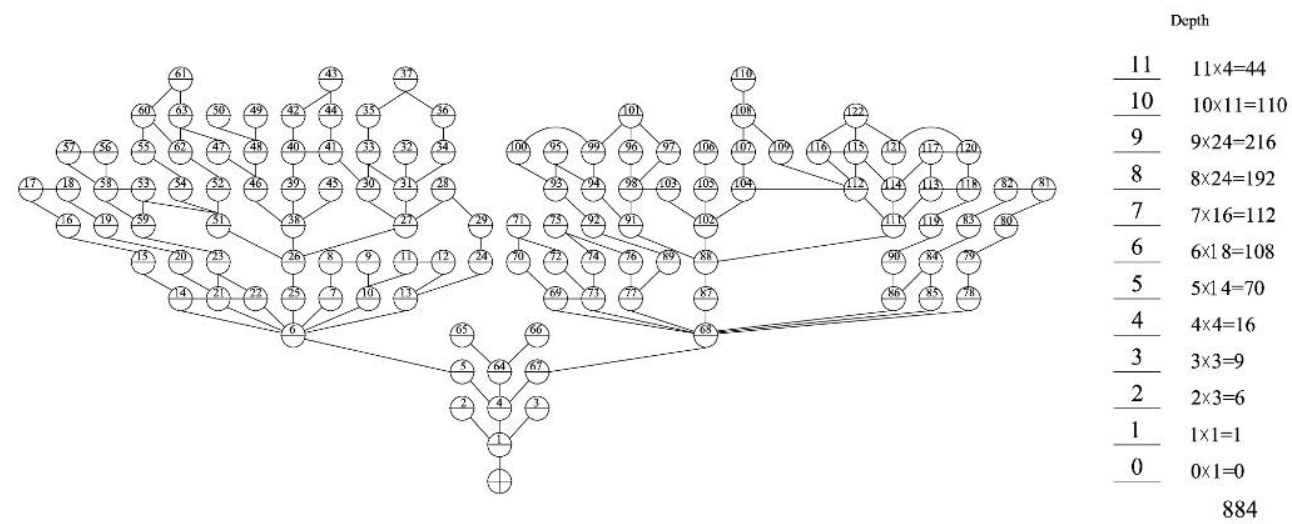

(a). Washington national gallery 1st floor topological depth map from outdoor

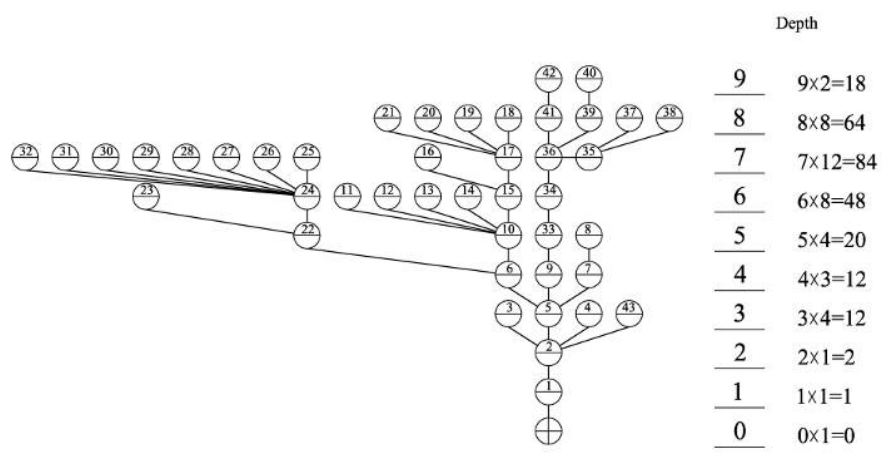

(b). Shanghai art gallery

1st floor topological depth map from outdoor

Figure 3: (a). the outdoor space as the starting point of J-graph, mean depth (MD) =7.25.

(b).the outdoor space as the starting point of J-graph, mean depth (MD) $=6.07$

\section{Comparison of convex integration}

Integration is a quantitative indicator that can describe the overall spatial structure, small radius value reflects the relationship between the spatial structures of local scale (such as walking movement), the large radius value reflects the overall spatial structures (such as car movement) ${ }^{[3]}$, in space syntax analysis software, the default radius value is 3 steps or step $n$ ( $\mathrm{n}$ is infinite). In a wide range of spatial scope, the relationship between these two integrations determines the spatial structure ranging from the entire elements to local parts. It controls the degree of movement of people on two levels. Relatively high spatial integration value constitutes the integration core. Typically more than 
$25 \%$ with high integration values within the space are chose to be the best integration area of the space and define the scope of integration core. Integration core usually connected building's particular important area, such as public areas via one or more directions to outer space, and is always combined with space in two ways: firstly, they are usually combined with the most important human movement pattern; secondly, space on behalf of major public function, such as residential living or the art gallery foyer, is usually combined with integrated core together (Hillier 1996) ${ }^{[5]}$.

Figure 3 (a) shows the integration core distribution of convex space of Washington national gallery, its mean integration core is 0.8794 and the average integration core value from $1^{\text {st }}$ floor to $3^{\text {rd }}$ floor of Shanghai art gallery respectively is $1.002,1.129$ and 1.049 (Fig. 3 (b)). With comparison we know that: mean integration core value of Washington national gallery is below that of the Shanghai art gallery from $1^{\text {st }}$ floor to $3^{\text {rd }}$ floor. Through field observation, visitor flow did not locate in the places where the highest integration value is, but scattered in several small compartments not far from the integration core, which again proves that integration core is not places where the most people always stay, but the places of the majority of people can see it. Therefore visibility is still the most important features of spatial integration.

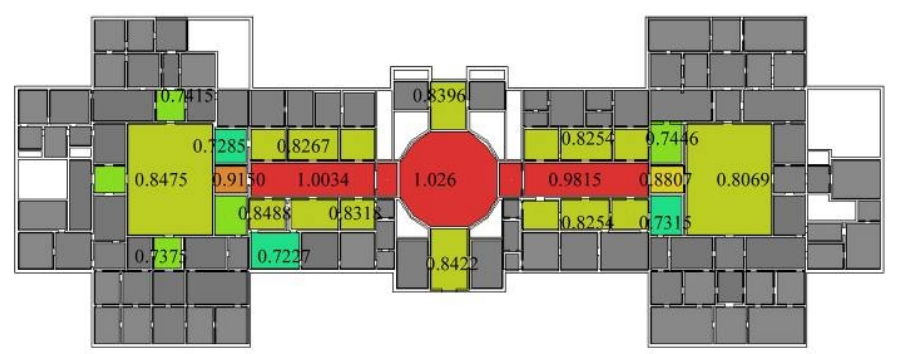

(a). Washington national gallery main floor
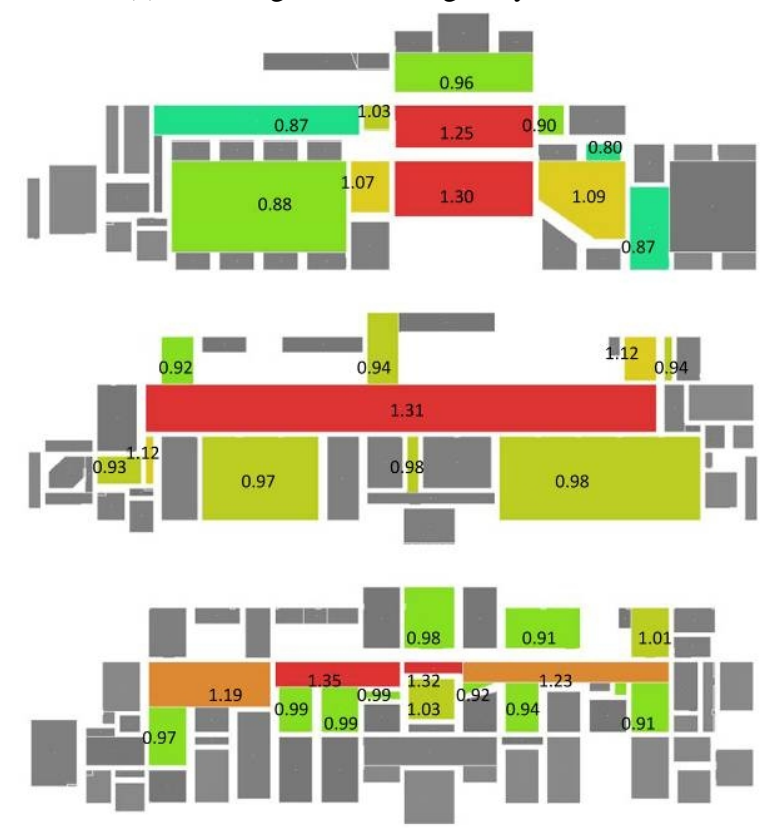

(b). Shanghai art gallery 1 st $\sim 3$ rd floor plan

Figure 4: convex space integration core

(representing more than $25 \%$ of the maximum degree of integration of the space)

Integration core reflects the position of the main public space, but also reflects the different movement patterns within the space and space usage condition of art gallery. Integration value has quite a close relationship with the size of neighboring space and the amount of being controlled space. Normally, the more connectivity of a space, the stronger conformity, but not necessarily the stronger integration value, it depends on the relationship between spatial configurations. Theoretically, integration core is a group of some convex spaces where people regularly gather, but according to Hillier and Choi's study ${ }^{[1]}$ : maximized random encounter is visualized within the space, or reaching visualization by increasing integration value. The pattern of visitor's presence do not directly related to the characteristics of space configuration between different art galleries, but it is closely related to 
the degree of integration, namely the integration core is not the most person staying places, but the places the most people can see it.

\section{Comparison of all axis line}

With respect to the convex analysis, all axis line map can reveal space configuraional relationship in more overall scope. Figure 5 (a) shows all axis line map of the main floor in Washington national gallery, which constitute a "H" shaped skeleton of sight by a group of the longest horizontal line of sight (the red line) across the hall from left end to right end and the secondary level of vertical line of sight (light blue line) in left and right sides of the hall, but most of the rest of the line of sight is short dark blue line, since each room is interrelated through the doorway, so the bottleneck effect have strengthened hierarchical structure of sight line, hierarchical structure of space sequence is coincident with the line of sight line, the shape of "H" frame of the sight line correspondingly coincide with the architectural plan, the convex space integration is compared with the all axis line integration, color distribution of red and blue position is almost the same, to be exact, the highly integrated of space displayed by these two methods is overlapped with each other; Compare to the above, the longest lines of sight from east end to west end (red line) also appear on both sides of the galleries of the 1st floor plan of Shanghai art gallery (Figure 6 (c)), most other lines of sight is relatively short blue line, if taking a close look, you will find the highest value of the integration distribution does not overlapped with the highest value of the connectivity distribution. Usually, the longest groups of line have the most chance to meet with other groups of line, so the highest value of the connectivity of axis is the lines from one end to another end (Figure 6 (c, up)), but the most integration axis is from lobby to two major exhibition hall (Figure 6 (c, down)), this phenomena is also can be observed through scatter plot, each axis line is represented by a scatter point, the shape of scatter points is like a fork that there are two major bands begin to separate in the middle value of integration, which means that there an another less stronger group of line exist on the plan, so totally 3 groups of high value integration line constitute the primary skeleton of triangle shape on the $1^{\text {st }}$ floor plan of Shanghai Art gallery.

Both architectures showed a symmetrical layout and the overall layout pattern are basically the same, but more rooms on the main floor of Washington national gallery and the spatial configurational relationship is more complex. Seemingly similar layout principle on the plan, but the spatially logical relationship has a slightly difference which can't be easily perceived by human initial perception, these quantitative tools and methods can be used to analyze and summarize the feature of a certain kind of space, in turn, it confirms to the conclusion of convex integration core by the method of convex analysis, that is the movement of integration core reflects the degree of overall understanding of floor layout. The integration core of convex space on the main floor is closer to outer space in Washington national gallery, which is easier to reach and identified, the visitors in the lobby can understand the general relationship between the overall spaces. However, the integration core of Shanghai art gallery is more likely located at center of the $1^{\text {st }}$ floor plan or at a little bit deeper places, in order to understand the overall spatial relationship, visitors need to locate on the passage just behind the lobby. 

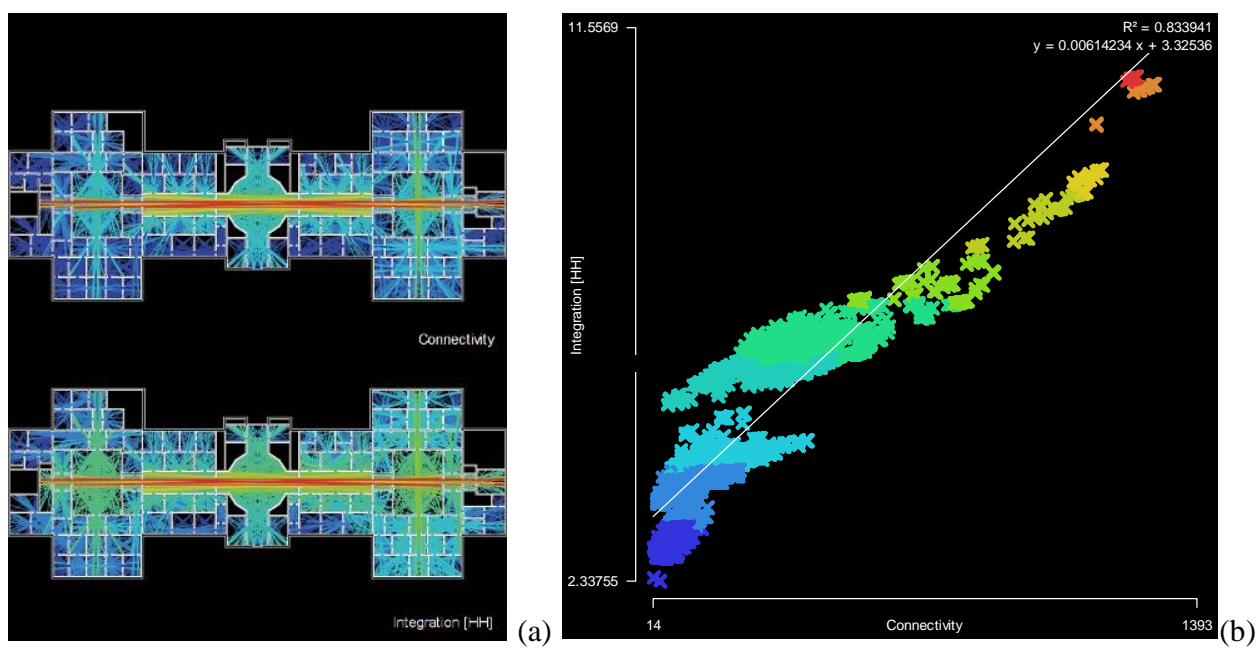

Figure 5: Washington national gallery main floor

(a).all axis line integration (b). connectivity-integration scatter plot
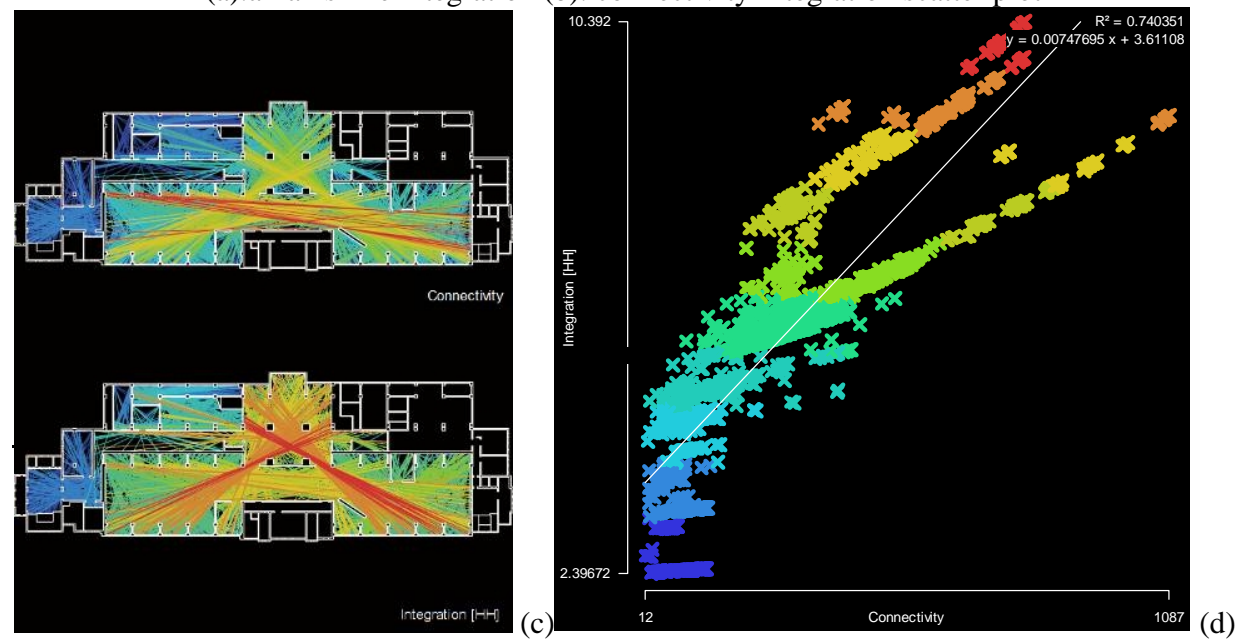

Figure 6: 1st floor of Shanghai art gallery

(c).all axis line integration (d). connectivity-integration scatter plot

\section{Conclusions}

The character of spatial configuration and quantified topology relationship of spatial configuration are calculated by the methods of space syntax with applying mathematics and statistical analysis, by comparison study, it can prove intuitive correctness and rationality of architects when they do design, reveal the exact value and some aspects of unnoticed spatial relationships, detail reveals the logical structure of interior space and how people use these spaces and meet in spaces. The advantages of its mathematical analysis are particularly apparent when the designer makes evaluation during process of design optimization and the building environment.

Variable space syntax analysis methods can accurately identify the space with high integration and better understandability position, "the feature of intelligibility means that, to what the extent do we see from a space could be useful guidance for what we can't see. The lack of intelligibility of systems are often not well integrated into the entire system, because there are many connections among spaces, these visible connections will mislead our understanding and intuitive perception in this whole space systems" ${ }^{[6]}$.

\section{References}

[1] Peponis, J., et al. (2015). "Configurational meaning and conceptual shifts in design." The Journal of Architecture 20(2): 215-243. 
[2] Tzortzi, K., The interaction between building layout and display layout in art gallerys, Ph.D. thesis, (University College London, UK.), 2007

[3] Peponis, J. (2012). "Building layouts as cognitive data purview and purview interface." Cognitive Critique, Volume 6: 11 51.

[4] Yoon Kyung Choi, The Spatial Structure of Exploration and Encounter in Art gallery Layouts, Ph.D. thesis, (Georgia Institute of Technology, USA.), 1991

[5] Hillier B, Space is the Machine. Cambridge University Press, 1996.

[6] Hsu Huang: The Spatialization of Knowledge and Social Relationships. Page:6-7 